\title{
Gamma-ray emitting supernova remnants as the origin of Galactic cosmic rays
}

\author{
M. Kroll, J. Becker Tjus, B. Eichmann, and N. Nierstenhöfer \\ Ruhr-Universität Bochum, Plasma Astroparticle Physics, 44780 Bochum, Germany \\ Correspondence to: M. Kroll (mike.kroll@ ruhr-uni-bochum.de)
}

Received: 13 May 2015 - Revised: 10 August 2015 - Accepted: 9 September 2015 - Published: 12 November 2015

\begin{abstract}
It is generally believed that the cosmic ray spectrum below the knee is of Galactic origin, although the exact sources making up the entire cosmic ray energy budget are still unknown. Including effects of magnetic amplification, Supernova Remnants (SNR) could be capable of accelerating cosmic rays up to a few PeV and they represent the only source class with a sufficient non-thermal energy budget to explain the cosmic ray spectrum up to the knee. Now, gamma-ray measurements of SNRs for the first time allow to derive the cosmic ray spectrum at the source, giving us a first idea of the concrete, possible individual contributions to the total cosmic ray spectrum. In this contribution, we use these features as input parameters for propagating cosmic rays from its origin to Earth using GALPROP in order to investigate if these supernova remnants reproduce the cosmic ray spectrum and if supernova remnants in general can be responsible for the observed energy budget.
\end{abstract}

1

\section{Introduction}

The origin of high energy cosmic rays (CR) remains uncertain, nevertheless supernova remnants are being promoted as the most promising candidates for providing the CR flux up to PeV energies measured at Earth (Fermi, 1949; Blandford and Ostriker, 1978; Hillas, 2005). Due to shock front acceleration in the surrounding dust cloud, charged particles are able to reach energies up to that order. Here a sudden break in the cosmic ray spectrum occurs, referred to as the knee.

The experiments H.E.S.S., MAGIC, VERITAS and MILAGRO have proven gamma rays from SNRs up to energies as high as $\sim 100 \mathrm{TeV}$. By additionally using $\mathrm{X}$-ray and radio data of those sources it can be identified whether hadronic or leptonic processes caused the very high energy (VHE) gamma rays. This differentiation has been conducted in Mandelartz and Becker Tjus (2015).

This approach allows the derivation of $j_{\mathrm{p}}(E)$, which gives information about a potential population of CR protons inside the SNRs. Since CR become deflected due to their electric charge by magnetic fields, and energy loss processes happen in interactions with Galactic gas clouds, it is impossible to directly extract information of those CRs regarding original direction and energy release by their SNR of origin.
Hence, this raises the importance of the indirectly determined parameter $j_{\mathrm{p}}(E)$.

On the contrary, gamma rays travel through the Galaxy nearly without being deflected, and their energy budget remains untouched by the Galactic environment.

In terms of propagating CRs through the Galactic disk to Earth we make use of the publicly available GALPROP code (Strong and Moskalenko, 1999). By applying certain modifications to this code we are able to inject CR nuclei of SNRs with spectra as derived in Mandelartz and Becker Tjus (2015).

However, the CR flux up to the knee will be underestimated when considering only today's observable SNRs as possible sources. This becomes clear, when comparing the average time of CRs travelling through the Galaxy to be about $10^{6}-10^{7}$ years to the lifetime of one SNR, which is about $10^{4}$ years, and thus significantly less.

We therefore take the spectra of all observable SNRs as a representative subsample of SNR spectra and luminosities. The time averaged sample of SNRs is obtained by simulating multiple SNRs at random positions in the Galactic disk, following the SNR distribution of Case and Bhattacharya (1998), which has an increased density near the Galactic center. Although this is the distribution we use in our analysis, 
there are other distribution that are considered to be more realistic, see e.g. Green (2014).

The primary objective of this proceeding is to describe the actual status of our analysis with special emphasis put on the overall normalization. We present an simulation that focuses on reproducing the slope of the proton flux measured at Earth. We do not present any optimization of model parameters, and all shown results are preliminarily ranked.

\section{Source spectra}

In Mandelartz and Becker Tjus (2015) the electromagnetic spectrum of 24 SNRs has been analyzed and the interaction type for generating VHE gamma rays, either leptonic or hadronic, has been identified. Sources with a very hard gamma ray spectra, e.g. $100 \mathrm{TeV}$ and above, are necessary to explain the knee in the CR spectrum. Young SNRs provide the best candidates. It is shown that 21 SNRs are causing VHE gamma rays through $\pi^{0}$-decay, hence characterized as a hadronic process. For further details see Table 3 in Mandelartz and Becker Tjus (2015).

In our analysis we choose these 21 SNRs as the source spectra $j_{\mathrm{p}}(E)$

$$
\begin{aligned}
j_{\mathrm{p}}(E) & =a_{\mathrm{p}}\left(\frac{E^{2}+2 E m_{\mathrm{p}} c^{2}}{E_{0}^{2}+2 E_{0} m_{\mathrm{p}} c^{2}}\right)^{-\frac{\alpha_{\mathrm{p}}}{2}} \frac{E+m_{\mathrm{p}} c^{2}}{\sqrt{E^{2}+2 E m_{\mathrm{p}} c^{2}}} \\
& \times \tanh \left(\frac{E}{E_{\text {min }, \mathrm{p}}}\right) \exp \left(-\frac{E}{E_{\text {max }, \mathrm{p}}}\right),
\end{aligned}
$$

with $E$ as the kinetic energy, $a_{\mathrm{p}}$ as the normalization in units of $\mathrm{MeV}^{-1}$, the proton mass $m_{\mathrm{p}}$, the normalization energy $E_{0}=1 \mathrm{TeV}$ as well as minimum and maximum energy $E_{\min }$ and $E_{\max }$, respectively. The minimum energy is fixed to a value of $E_{\min }=10 \mathrm{MeV}$ while the maximum energy depends on the actual SNR. See Table 3 in Mandelartz and Becker Tjus (2015) for detailed information on that energy.

Equation (1) represents a power law in momentum space with low- and high energy cut-offs. Since we analyze cosmic ray energies in the range from $\sim \mathrm{GeV}$ to $\sim \mathrm{PeV}$ the low energy cutoff is negligible.

The Greens catalogue (Green, 2009) contains a total number of 274 SNRs, whereas only about ten percent have been observed in gamma rays. Hence, by only taking those SNRs into account, we expect a too low prediction of the CR flux.

\section{Propagation}

The source spectra $j_{\mathrm{p}}(E)$ as parametrized in Eq. (1) is propagated through the Galactic disk with the open source $\mathrm{C}++$ tool GALPROP (Strong et al., 2012). In order to perform this, certain modifications have to be applied to the code. Secondly, a function has been implemented that selects a random spatial position of the SNRs, following the SNR distribution as presented in Case and Bhattacharya (1998).

\subsection{Normalization}

The CR normalization as implemented in standard GALPROP routines is adjusted to the observed CR flux at Earth. This is obtained by a global scale factor of the calculated CR density. Our approach requires to determine the normalization by the rate of particle injection of individual SNRs to the Galaxy. The source spectrum $j_{\mathrm{p}}(E)$ must be rewritten in terms of internal GALPROP units. The normalization factor will therefore be determined by the ratio of GALPROP's calculated luminosity $L_{\mathrm{G}}$ with the corresponding integral expression

$L=\alpha R_{\mathrm{SNR}}^{2} c \int_{10 \mathrm{MeV}}^{1 \mathrm{PeV}} \mathrm{d} E \beta E j_{\mathrm{p}}(E)$.

In the above equation, $R_{\mathrm{SNR}}$ is the radius of the SNR. With $\alpha=1$ the emitted $\mathrm{CR}$ exit the source in radial directions, while the case $\alpha=1 / 2$ considers random directions of CRs inside the SNR.

The relationship between the initial source function $q_{1}(p(E))$ that GALPROP uses and our parametrization is

$q_{1}(p(E))=\alpha \frac{c^{2} R_{\mathrm{SNR}}^{2} \beta^{2}}{4 \pi V_{\text {grid }}} j_{\mathrm{p}}(E)$.

As a second approach, the total energy of protons $E_{\text {tot,p }}$ gives information about the luminosity, as well. Taking a timescale factor $\tau$ the average luminosity of CRs $L=E_{\text {tot,p }} / \tau$ represents the distribution of the total energy over the total lifetime of the remnant,

$L=\frac{1}{\tau} \frac{4 \pi}{3} R_{\mathrm{SNR}}^{3} \int_{10 \mathrm{MeV}}^{1 \mathrm{PeV}} \mathrm{d} E E j_{\mathrm{p}}(E)$.

Hence, Eq. (3) transforms to

$q_{1}^{\prime}(p(E))=\beta \frac{c^{2} R_{\mathrm{SNR}}^{3}}{3 \tau V_{\text {grid }}} j_{\mathrm{p}}(E)$.

In the following paragraph we show that the average CR spectrum can be reproduced by simplifying assumptions. In particular, the assumptions include that all SNR have (a) the same spectral index, (b) the same energy budget and (c) the same maximum energy.

The released energy budget of a standard supernova explosion can be estimated to about $E_{\mathrm{SN}}=10^{51} \mathrm{erg}$. Considering the assumption that the CR flux below the knee represents the CR content of the Galaxy we can estimate within an order of magnitude uncertainty the CR luminosity to $L \sim 2 \times 10^{41} \mathrm{erg} \mathrm{s}^{-1}$, see e.g. O’C. Drury (2014).

The rate of supernova explosions is approximately $\dot{n} \approx 1 / 50 \mathrm{yr}^{-1}$. Additionally, we can motivate a constant factor $\eta$, which stands for the fraction of kinetic energy that converts to hadronic cosmic rays. 
Already $\eta=10 \%$ is able to explain the total cosmic ray energy budget,

$L_{\mathrm{CR}} \approx 2 \times 10^{41}\left(\frac{\eta}{0.1}\right)\left(\frac{\dot{n}}{0.02 \mathrm{yr}^{-1}}\right)\left(\frac{E_{\mathrm{SN}}}{10^{51} \mathrm{erg}}\right)$.

This back-of-an-envelope calculation demonstrates that the average CR spectrum provided by SNRs is indeed met by a few simplifying but reasonable assumptions. This claim, however, has not been proven by using actual SNR cosmic ray spectra. Especially, a more elaborate approach would include not only a time-dependent energy spectrum but also a incorporate the reduction of the maximum energy throughout time, see e.g. Cox (1972).

Also in Cox (1972), cooling effects are responsible for a decrease of the total energy. This behavior would certainly effect the average luminosity of CRs, and hence our assumption of a constant fraction $\eta$ would overestimate the luminosity most notably for old SNRs.

Nevertheless, despite the aforementioned discussion, we believe in an first order approximation that our calculation is reasonable. The presented results in Sect. 4, however, do not use these simplifying assumptions but uses SNR parameters as derived in Mandelartz and Becker Tjus (2015).

\subsection{Cosmic ray nuclei}

GALPROP allows to simulate protons but also CR nuclei of higher atomic numbers can be included to the calculation routine.

The relative abundance to the initial source function of protons is defined as

$X=\frac{A \cdot q_{A}\left(p_{A}\right)}{q_{1}\left(p_{1}\right)}$,

where $p_{A}$ denotes the momentum of the nuclei with mass number $A$.

The energy cutoff in Eq. (1) is high enough that the energy dependence of the relative abundance is negligible and therefore not included in further analyses. Technically, as for $q_{A}$ we have used the GALPROP standard value.

There is one technical side effect that occurs by adding higher order nuclei to the simulation. The total energy of hadrons in SNRs artificially increases due to that addition. However, this can easily be counteracted by downscaling the proton normalization in Eq. (1), which virtually corrects the energy budget of the spectrum taken from Mandelartz and Becker Tjus (2015).

The present analysis shows simulations of all available nuclei, however, the energy spectrum as shown below is the result of simulating protons only. As a future prospect, simulations of heavy CR nuclei will be helpful when facing questions regarding cosmic ray observations, analyses of CR sources as well as the transport of these particles.

\subsection{GALPROP settings}

The key concept of GALPROP simulations is to slice the Galaxy into a grid. Each grid point evaluates the incoming flux and propagates it to the next one. In our case, the Galaxy is treated as three dimensional body, and all grid points are equidistant to one another with $d_{\text {grid }}=1 \mathrm{kpc}$. The large distance allows to treat the SNRs as point sources because their radius is significantly smaller.

The physical size of the Galaxy in the horizontal plane is set to $x, y \in(-10,10) \mathrm{kpc}$, and vertically it ranges $z \in(-4,4) \mathrm{kpc}$. This way, we can prove our computational ability and it gives a first estimate on our preliminary results.

Besides that, we have used basic parameters as suggested by the GALPROP web-run (Strong et al., 2012).

\section{Exemplary simulation}

In this section we show our preliminary results. We have used $N=100$ sets of 21 SNRs distributed randomly in the Galactic grid. Throughout this proceeding the notation $\langle\ldots\rangle$ stands for the average of $N=100$ simulations so that the simulation is comparable to experimental observations. Moreover, we refer to the kinetic energy as used in above equations with the letter $T$.

Generally we have estimated the lifetime of one SNR, where its CR radiation is relevant, to $T_{\mathrm{SNR}}=10^{4}$ years, and in each run the SNRs are all lit up all at once. Figure 1 shows a selection of the simulated $N=100$ GALPROP runs with 21 SNRs each, along with its average $<\mathrm{d} F / \mathrm{d} T>$. Furthermore, it has been normalized to CREAM (Yoon et al., 2011) data at $3.25 \mathrm{TeV}$ in order to better compare its slope to the data. Also, the solar potential has a value of $550 \mathrm{MV}$. In this plot, the yellow band represents the statistic variance over all simulations. That is, the mean value of all runs has been allowed to have an $1 \sigma$ uncertainty.

As shown in Fig. 1 the spectral behavior is described very well for energies higher than $E=1 \mathrm{TeV}$. PAMELA (Adriani et al., 2011) data, on the contrary shows a different slope than predicted by our simulation.

Since we derive the CR spectrum from gamma ray measurements we expect a best match of data in the range of $1-100 \mathrm{GeV}$ for Fermi and $0.1-10 \mathrm{TeV}$ for IACTs. Our assumption of $\eta=10 \%$ of CR energy is converted in photons, be expect reasonably good agreement in a range of $10 \mathrm{GeV}$ to $100 \mathrm{TeV}$. Extrapolation effects do not cause this discrepancy.

The Boron over Carbon ratio serves as a cross check and is shown in Fig. 2. It can be seen that the simulation with heavier nuclei includes reproduces AMS-01 data well. More importantly, this cross check proves that the number of SNRs indeed provides a high enough CR flux to match the measured data. The spectral behavior has here a minor contribution. 


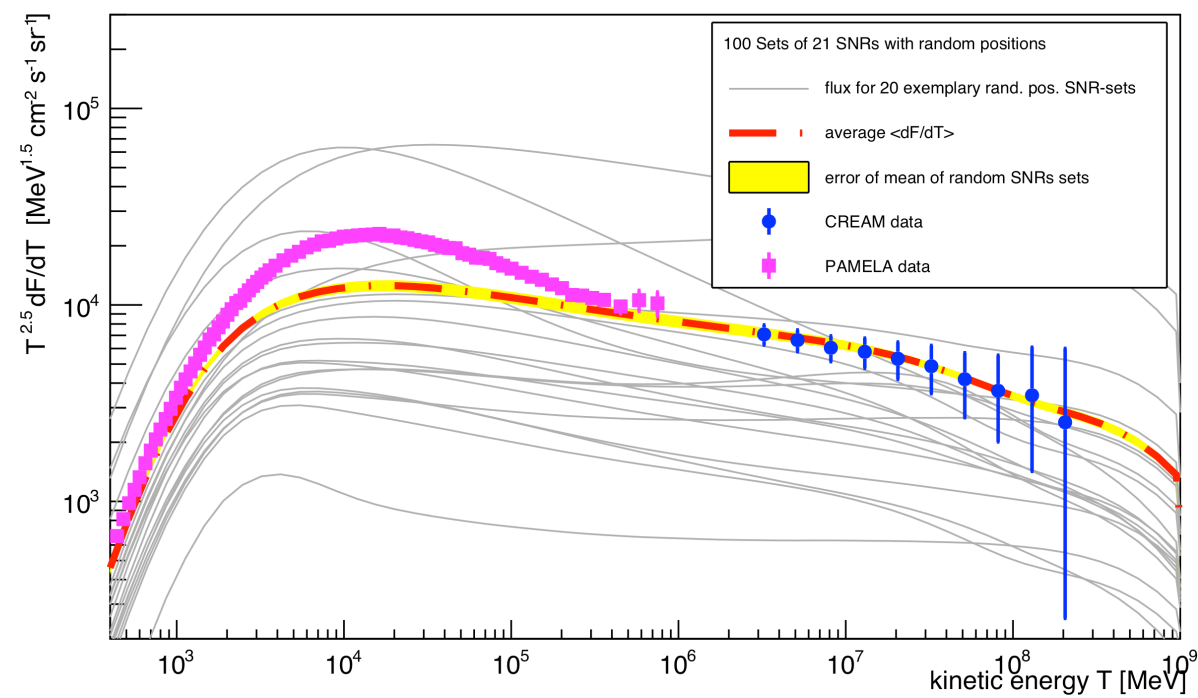

Figure 1. This figure shows 20 (solid grey) out of a total number of 100 simulated proton spectra, all containing the 21 SNRs presented in Mandelartz and Becker Tjus (2015). The mean $<\mathrm{d} F / \mathrm{d} T>$ (red, dashed line) and its error (solid, yellow) are shown as well. Experimental data are taken from CREAM (Yoon et al., 2011) and PAMELA (Adriani et al., 2011). The mean has been normalized to the lowest energy data point by CREAM.

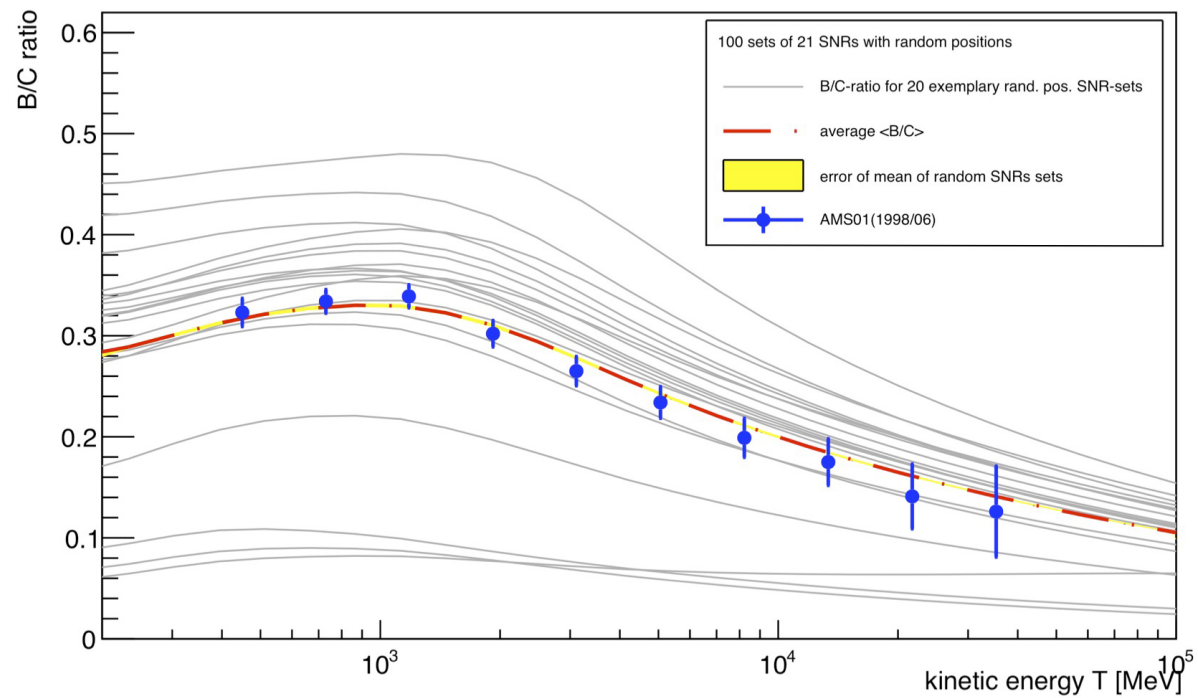

Figure 2. This figure shows 20 (solid grey) out of a total number of 100 simulated $\langle B / C>$ ratios, all containing the 21 SNRs presented in Mandelartz and Becker Tjus (2015). The mean, $\langle B / C\rangle$ (red, dashed line) and its error (solid, yellow) are shown as well. Data has been taken from the AMS-01 experiment (AMS-01 Collaboration, 2010).

\section{Discussion}

Especially Fig. 2 proves very good agreement with experimental data. This benchmark test is a first supporting argument for our simulation method. However, the discrepancies to PAMELA data of our averaged flux are evident, and thus, possible reasons for it shall be discussed in this section.

At first, statistics may cause an issue in our analysis. We can find an average of the whole set of simulations, $<\mathrm{d} F / \mathrm{d} T>$, but the variance is not to be determined. This measure is important to decide whether or not statistic parameters cause the failure at lower energies. Of course, the variance of $\mathrm{CR}$ observables may be presented here, but its statistical interpretation is limited. The variance would only represent the spread of CR observables, which is induced by randomly selected positions of the 21 SNRs, hence, decreasing behavior is expected when more SNRs are considered.

By putting more emphasis on time during the simulation, i.e. production rate or lifetime, the then calculated variance would be physically more meaningful. The GALPROP man- 
ual gives hints on how this could be implemented and will be investigated soon.

Furthermore, a close-by source that happens to coincide with one of the Earth's closest grid points would sophisticate the entire calculation as well, particularly when rather few simulations are performed.

Another aspect regarding numerical stability arises when simulating SNR as CR sources as $\delta$-functions. Despite the fact that sharp decreases in spatial source distributions are an uneasy way of treatment in numerical simulations, all our simulations seem to remain stable when variations of numerical time steps are considered.

Secondly, physical issues shall be taken into account.

In the current configuration the Kolmogorov diffusion scalar coefficient is used with value of $\alpha_{\text {diff }}=0.33$. This coefficient is worth investigating as it directly affects the steepening of the spectrum. There might be a value that reproduces low energy data better than the actual one, see e.g. Obermeier et al. (2012).

This behavior could be explained by missing SNRs with a flatter spectrum that have not yet been observed. With a higher sensitivity experiments like HAWK and CTA it may be possible to retrieve a more complete sample of SNRs. Other experiments like IceCube and KM3NET could resolve the parts of the gamma ray signal that hadronizes, see e.g. Becker et al. (2011), Schuppan et al. (2012) and Schuppan et al. (2014).

A further aspect would be to consider the scalar diffusion as a broken power law; the kink would occur at $\sim 100 \mathrm{GeV}$, as already pointed out by Strong et al. (2012).

With unreached accuracy the diffusion process is presented in a tensor structure. Naturally, this opens a complex field of studies but first steps have been already taken and been presented in publications using GALPROP's successor DRAGON for CR propagation and the PICARD code, see e.g. Gaggero et al. (2013) and Kissmann (2014).

All the above mentioned aspects might lead to a better description of data by our analysis. First indications of a verification of our method already exist.

\section{Conclusions}

In this proceeding we have introduced a procedure to predict the CR flux measured at Earth up to the knee. The tool for propagating CR particles from their source to Earth, i.e. GALPROP, allows to simulate protons as well as heavier nuclei. As possible sources for explaining the CR spectrum we investigated SNRs that feature a hadronic part in their spectrum (Mandelartz and Becker Tjus, 2015). In terms of simulation, we distributed the SNRs randomly on grid points, which is how the GALPROP code is structured, according to a given SNR distribution function.

The obtained results with this method show that our proton flux $<\mathrm{d} F / \mathrm{d} T>$ differs in slope in the low energy regime, for $E<10^{6} \mathrm{MeV}$, with data. However, for higher energies as well as for $\langle B / C>$ ratio we find a good agreement with data.

Our objective of this proceeding was to introduce our approach along with its techniques. As there is still a lot of work in progress, we are not able to present a parameter optimization nor do we want to draw far-reaching conclusions. Especially the normalization of the spectrum requires further effort as this will give definite answers wether SNRs with a hadronic part in their gamma ray spectrum are capable of explaining the observed CR flux.

Our future plans cover adding temporal features to the simulation in order to enhance the quality of our statistics, including more suitable SNRs when observed by experiments with higher sensitivities, and also implementing an inhomogeneous grid through the Galaxy.

Monte-Carlo simulations can be performed, as well, that will propagate the CR through the Galactic medium. One approach would be the also publicly available code CRPropa (Kampert et al., 2013). First estimations have been made to analyze Galactic CR of kinetic energies $E \in(10,100) \mathrm{TeV}$ with these Monte-Carlo techniques and calculation times are considered reasonable. Lower energies might require to use a diffuse approximation instead, since solving the equations of motion it that case turns out be extraordinary time consuming.

Acknowledgements. We acknowledge support from the research unit FOR1048 "Instabilites, Turbulence and Transport in Cosmic Magnetic Fields", as well as from the Research Department of Plasmas with Complex Interactions (Bochum) and the MERCUR Ruhr Astroparticle Plasma Physics Center (RAPP Center).

N. Nierstenhöfer would like to thank A. W. Strong and C. Evoli for insights about the GALPROP code. Furthermore we would like to thank D. Green and I. Gebauer for fruitful discussions.

Edited by: P. Desiati

Reviewed by: I. Gebauer and another anonymous referee

\section{References}

Adriani, O., Barbarino, G. C., Bazilevskaya, G. A., Bellotti, R., Boezio, M., Bogomolov, E. A., Bonechi, L., Bongi, M., Bonvicini, V., Borisov, S., Bottai, S., Bruno, A., Cafagna, F., Campana, D., Carbone, R., Carlson, P., Casolino, M., Castellini, G., Consiglio, L., De Pascale, M. P., De Santis, C., De Simone, N., Di Felice, V., Galper, A. M., Gillard, W., Grishantseva, L., Jerse, G., Karelin, A. V., Koldashov, S. V., Krutkov, S. Y., Kvashnin, A. N., Leonov, A., Malakhov, V., Malvezzi, V., Marcelli, L., Mayorov, A. G., Menn, W., Mikhailov, V. V., Mocchiutti, E., Monaco, A., Mori, N., Nikonov, N., Osteria, G., Palma, F., Papini, P., Pearce, M., Picozza, P., Pizzolotto, C., Ricci, M., Ricciarini, S. B., Rossetto, L., Sarkar, R., Simon, M., Sparvoli, R., Spillantini, P., Stozhkov, Y. I., Vacchi, A., Vannuccini, E., Vasilyev, G., Voronov, S. A., Yurkin, Y. T., Wu, J., Zampa, 
G., Zampa, N., and Zverev, V. G.: PAMELA Measurements of Cosmic-Ray Proton and Helium Spectra, Science, 332, 69-72, doi:10.1126/science.1199172, 2011.

AMS-01 Collaboration: Relative Composition and Energy Spectra of Light Nuclei in Cosmic Rays, Results from AMS-01, ArXiv e-prints, 2010.

Becker, J. K., Black, J. H., Safarzadeh, M., and Schuppan, F.: Tracing the Sources of Cosmic Rays with Molecular Ions, Astrophys. J. Lett., 739, L43, doi:10.1088/2041-8205/739/2/L43, 2011.

Blandford, R. D. and Ostriker, J. P.: Particle acceleration by astrophysical shocks, Astrophys. J. Lett., 221, L29-L32, doi:10.1086/182658, 1978.

Case, G. L. and Bhattacharya, D.: A New $\Sigma$-D Relation and Its Application to the Galactic Supernova Remnant Distribution, Astrophys. J., 504, 761-772, doi:10.1086/306089, 1998.

Cox, D. P.: Cooling and Evolution of a Supernova Remnant, Astrophys. J., 178, 159-168, doi:10.1086/151775, 1972.

Fermi, E.: On the Origin of the Cosmic Radiation, Phys. Rev., 75, 1169-1174, doi:10.1103/PhysRev.75.1169, 1949.

Gaggero, D., Maccione, L., Di Bernardo, G., Evoli, C., and Grasso, D.: Three-Dimensional Model of Cosmic-Ray Lepton Propagation Reproduces Data from the Alpha Magnetic Spectrometer on the International Space Station, Phys. Rev. Lett., 111, 021102, doi:10.1103/PhysRevLett.111.021102, 2013.

Green, D. A.: A revised Galactic supernova remnant catalogue, Bull. Astron. Soc. India, 37, 45-61, 2009.

Green, D. A.: The Galactic distribution of SNRs, in: IAU Symposium, vol. 296 of IAU Symposium, edited by: Ray, A. and McCray, R. A., 188-196, doi:10.1017/S1743921313009459, 2014.

Hillas, A. M.: Can diffusive shock acceleration in supernova remnants account for high-energy galactic cosmic rays?, J. Phys. G, 31, R95-R131, 2005.

Kampert, K.-H., Kulbartz, J., Maccione, L., Nierstenhoefer, N., Schiffer, P., Sigl, G., and van Vliet, A. R.: CRPropa $2.0-$ A public framework for propagating high energy nuclei, secondary gamma rays and neutrinos, Astropart. Phys., 42, 41-51, doi:10.1016/j.astropartphys.2012.12.001, 2013.

Kissmann, R.: PICARD: A novel code for the Galactic Cosmic Ray propagation problem, Astropart. Phys., 55, 37-50, doi:10.1016/j.astropartphys.2014.02.002, 2014.
Mandelartz, M. and Becker Tjus, J.: Prediction of the diffuse neutrino flux from cosmic ray interactions near supernova remnants, Astropart. Phys., 65, 80-100, doi:10.1016/j.astropartphys.2014.12.002, 2015.

Obermeier, A., Boyle, P., Hörandel, J., and Müller, D.: The Boronto-carbon Abundance Ratio and Galactic Propagation of Cosmic Radiation, Astrophys. J., 752, 69-76, doi:10.1088/0004637X/752/1/69, 2012.

O'C. Drury, L.: Origin(s) of Cosmic Rays, ArXiv e-prints, 2014.

Schuppan, F., Becker, J. K., Black, J. H., and Casanova, S.: Cosmicray-induced ionization in molecular clouds adjacent to supernova remnants, Tracing the hadronic origin of $\mathrm{GeV}$ gamma radiation, Astron. Astrophys., 541, A126, doi:10.1051/00046361/201218849, 2012.

Schuppan, F., Röken, C., and Becker Tjus, J.: Theoretical study of ionization profiles of molecular clouds near supernova remnants. Tracing the hadronic origin of $\mathrm{GeV}$ gamma radiation, Astron. Astrophys., 567, A50, doi:10.1051/0004-6361/201423614, 2014.

Strong, A. W. and Moskalenko, I. V.: New constraints on Galactic cosmic-ray propagation, http://galprop.stanford.edu, last access: 19 October 2015, 279-282, 1999.

Strong, A. W., Moskalenko, I. V., Porter, T. A., Gudlaugur, J., Orlando, E., Digel, S. W., and Vladimirov, A. E.: GALPROP Homepage, http://galprop.stanford.edu (last access: 19 October 2015), 2012.

Yoon, Y. S., Ahn, H. S., Allison, P. S., Bagliesi, M. G., Beatty, J. J., Bigongiari, G., Boyle, P. J., Childers, J. T., Conklin, N. B., Coutu, S., DuVernois, M. A., Ganel, O., Han, J. H., Jeon, J. A., Kim, K. C., Lee, M. H., Lutz, L., Maestro, P., Malinine, A., Marrocchesi, P. S., Minnick, S. A., Mognet, S. I., Nam, S., Nutter, S., Park, I. H., Park, N. H., Seo, E. S., Sina, R., Swordy, S., Wakely, S. P., Wu, J., Yang, J., Zei, R., and Zinn, S. Y.: Cosmic-ray Proton and Helium Spectra from the First CREAM Flight, Astrophys. J., 728, 122-130, doi:10.1088/0004-637X/728/2/122, 2011. 